\title{
La exploración y explotación de gas en lutitas como un proceso de (in)sustentabilidad en Tamaulipas
}

\section{Edith Miriam García Salazar}

Catedrática Conacyt -Colegio del Estado de Hidalgo SNI C, mgarcia@elcolegiodehidalgo.edu.mx Recibido: 15-11-18 Aceptado: 19-02-2019 Publicado: 15-08-2019

Cómo citar este artículo: García M. (2019) "La expplración y explotación de gas en lutitas como un proceso de (in)sustentabilidad en Tamaulipas" en Revista Científica de Estudios Urbano Regionales Hatsö-Hnini, Año 1, Núm. 1, Abril-Septiembre 2019, págs. 58-71 México

DOI: https://doi.org/10.47386/2019V1N1A5

\section{Resumen}

La profundización de los problemas ambientales derivados de la explotación acelerada de los recursos naturales, en combinación con los procesos de rápida industrialización, han traído como consecuencia una pérdida de bienestar y de calidad de vida al grueso de la población. Este hecho se ve reflejado en un deterioro de la salud humana, el hambre, la toxicidad, así como del cambio climático, entre otros. A raíz de ello, durante la década de los setenta surge el concepto de desarrollo sustentable como una estrategia para la concientización y uso racional de los recursos naturales, a partir de una nueva relación entre la naturaleza y la sociedad, para el goce de las generaciones futuras.

El presente documento aborda como tema central los efectos negativos que la exploración y explotación de gas en lutitas podrían generar en el estado de Tamaulipas ante la utilización de la técnica del fracking, y las repercusiones ambientales derivadas de cambios en la utilización de recursos provenientes de los mantos acuíferos de la entidad, así como otros efectos nocivos en el aire y el suelo. Se parte del supuesto de que la implementación de dicha técnica traerá como consecuencia, problemas de sustentabilidad, principalmente en el caso del agua. Por lo consiguiente, es a partir de la visión de desarrollo sustentable que se abordan las implicaciones ambientales del uso de esta técnica.

El trabajo se divide en cuatro partes, en la primera se mencionan de manera concisa los antecedentes de la actividad de la industria petrolera en torno a la producción de gas en lutitas. En la segunda parte, se señala de manera breve el desarrollo del concepto de desarrollo sustentable, como una guía para analizar las implicaciones en la sustentabilidad del estado de Tamaulipas. En la tercera parte, se analizan los aspectos sociodemográficos y territoriales en Tamaulipas, en los cuales se identifican las implicaciones ambientales del uso del fracking. En la cuarta parte, se enuncian las implicaciones en la sustentabilidad de Tamaulipas tomando como base las ideas de diversos autores en torno al concepto de sustentabilidad. [1] Finalmente, se presentan las conclusiones que derivan de este estudio.

\section{Antecedentes en torno al gas en lutitas}

Durante los últimos años, la baja de reservas de hidrocarburos en nuestro país ha conducido a la búsqueda de energías alternativas como aquellas contenidas en gas en lutitas [2], mismas que con la llegada de mejoras en las tecnologías para la extracción, han abierto la oportunidad de mostrar su rentabilidad en el mercado de hidrocarburos. En este sentido y de acuerdo con datos de la U.S. Energy Information Administration (U.S. EIA), México cuenta con potencial para exploración y explotación de este tipo de gas, ocupando el sexto lugar en el ranking mundial con una posible explotación de 545 billones de pies cúbicos, de los cuales, Tamaulipas es el estado 
con mayores reservas recuperables en el país, aproximadamente un 63 por ciento se encuentran en provincias petroleras relacionadas con el estado -TampicoMisantla y Burgos- (Gobierno del Estado de Tamaulipas, s.f., p. 10).

Las posibles reservas recuperables de gas en lutitas en Tamaulipas y las implicaciones ambientales y sociales de su exploración y explotación, llevan a cuestionar la posibilidad de si ello llevará a generar un desarrollo sostenible en el estado, o bien, si ello provocará una (in)sustentabilidad con graves repercusiones en la calidad de vida de la población y el deterioro de los ecosistemas. Si bien el discurso político que se ha manejado en torno a la participación privada en la industria se encuentra centrado en enumerar los aparentes beneficios económicos que ésta traerá al país y, para el presente estudio, en el caso de Tamaulipas, la ausencia de estudios científicos sobre temas de igual o mayor importancia tales como los posibles efectos negativos en el ámbito social (salud) y ambiental (afectaciones en el uso del agua, contaminación en el aire y afectaciones en el suelo), han caracterizado al discurso gubernamental al punto de que éstos no aparecen de manera explícita o como temas centrales dentro de la agenda de gobierno. Entre ellos se puede destacar el referido a uso de la técnica de fracturación hidráulica o fracking, la cual, y tal y como se ha documentado en diversos estudios, requiere de la inyección de grandes volúmenes de agua, arena, y de otros agentes químicos a alta presión para extraer el gas contenido en las rocas, por lo que pueden generarse afectaciones importantes en el medio ambiente.
En el siguiente apartado se menciona de manera breve las difer entes conceptualizaciones del desarrollo sustentable para a través de ello analizar el caso de estudio de Tamaulipas.

\section{Conceptualización del desarrollo sustentable}

Hablar de desarrollo sustentable remite de manera inmediata a la definición generada después de la realización de la conferencia de Estocolmo en el año de 1987, en la cual se crean el Programa de Naciones Unidas para el Medio ambiente y la Comisión Mundial sobre el Medio Ambiente y el Desarrollo. Y es a través de la Comisión Brundtland en el in $f$ o $r$ me Nuestro $F$ u tu $r$ o Común publicado en 1987 que se define como la "satisfacción de las necesidades de la generación presente sin comprometer la capacidad de las generaciones futuras para satisfacer sus propias necesidades", y se establece que la pobreza, la igualdad y la degradación ambiental no pueden ser analizados de manera aislada. En el documento se coloca a la pobreza como una de las causas de la degradación ambiental (Foladori y Tommasino, 2000, p. 44; Martínez-Alier, 1992, pp. 90 y 106), señala que la definición implícita de desarrollo sustentable es "crecimiento o desarrollo económico que sea compatible con la capacidad d e sustentación".

El concepto de desarrollo sustentable tiene sus raíces en la concepción del ecodesarrollo, el cual fue planteado por

I. Sachs, y comenzó a gestarse en la reunión de la ONU-EPHE previa a la - 


\section{La explotación y exploración de gan en lutitas}

Conferencia de las Naciones Unidas sobre el Medio Ambiente Humano en Estocolmo en el año de 1972. Sachs (1980,pp. 719 y 720$)$ define el ecodesarrollo como "desarrollo social, viable desde el punto de vista económico y prudente desde el ecológico [...] ofrece un criterio de racionalidad social diferente de la lógica de mercado, que se basa en los postulados éticos complementarios de la solidaridad sincrónica con la generación actual y de la solidaridad diacrónica con las generaciones futuras". Durante la reunión realizada en Estocolmo se tuvo como resultado una "declaración donde se abordaron los principales problemas relacionados con el medio ambiente: industrialización, explosión demográfica y crecimiento urbano. Se proclamó el derecho de los seres humanos a un ambiente sano y el deber de protegerlo y mejorarlo para las futuras generaciones" (Foladori y Tommasino, 2000, p. 43).

La conferencia de Estocolmo marco el antes y el después de la publicación los Limits of Growth en 1972, en la cual Meadows et al. (1972, p. 3) enunciaban que "si se mantienen las tendencias actuales de crecimiento de la población mundial, industrialización, contaminación ambiental, producción de alimentos y agotamiento de los recursos, este planeta alcanzará los límites de su crecimiento en el curso de los próximos cien años. El resultado más probable sería un súbito e incontrolable descenso tanto de la población como de la capacidad industrial". Los precursores de este informe son el grupo conocido como el Club de Roma, marcando con esto por primera vez en la historia la crisis ecológica que afecta al planeta, y la cual es ocasionada por el ser humano.
Es desde estas perspectivas que el concepto de desarrollo sustentable se aplica en la formulación de las políticas dirigidas al mejoramiento ambiental, pero que sucede en el caso del estado de Tamaulipas y la reciente apertura a la exploración y explotación de hidrocarburos en el país. A partir, de lo anterior se analiza el de Tamaulipas, esto como consecuencia de un e ncaminamiento hacia la (in)sustentabilidad [3] derivado de los efectos negativos en el ambiente por el uso de la técnica del fracking.

\section{Tamaulipas.Aspectos sociodemográficos y territoriales}

El estado de Tamaulipas se localiza al norte $27^{\circ} 40^{\prime}$, al sur $22^{\circ} 12^{\prime}$ de latitud norte; al este $97^{\circ} 08^{\prime}$, al oeste $100^{\circ} 08^{\prime}$ de longitud oeste. Colinda al norte con el estado de Nuevo León y Estados Unidos de América; al este con Estados Unidos de América y el Golfo de México; al sur con el Golfo de México y los estados de Veracruz-Llave y San Luis Potosí; al oeste con los estados de San Luis Potosí y Nuevo León. Representa el 4.1 por ciento de la extensión territorial total del país. El estado tiene 43 municipios que se dividen en seis regiones: 1) Región Fronteriza, 2) Valle de San Fernando, 3) Centro, 4) Altiplano, 5) Mante, y 6) Sur. La población total del estado, de acuerdo con el Censo de Población y Vivienda 2010, es de 3,268,554 habitantes, de la población mayor a 14 a ños e 160.1 por ciento es económicamente activa para el segundo trimestre del año 2014. 


\section{La exploración y explotación de gas en lutitas}

El estado localizado al noreste del país tuvo una aportación de 3.1 por ciento al Producto Interno Bruto (PIB) de la economía nacional en 2015. De éste, 2.9 corresponde al desarrollo de actividades primarias (agricultura, ganadería, aprovechamiento forestal, pesca y caza), 38.6 a actividades secundarias (minería; construcción y electricidad; agua y gas; e industrias manufactureras), y 58.5 por ciento a actividades tercerías (comercio, servicios de alojamiento temporal y de preparación de alimentos y bebidas, transportes e información en medios masivos, servicios financieros e inmobiliarios, servicios educativos y médicos, actividades del gobierno y otros servicios). Por su parte, el índice de desarrollo humano (IDH) registró valores de $0.846,0.637,0.809$ para los rubros de salud, educación e ingreso respectivamente, mientras que el global del estado fue de 0.758 en 2012 (PNUD, 2015), cifra comparable con niveles de desarrollo de países como Trinidad y Tobago y Antigua y Barbuda (PNUD, 2014). Estos indicadores económicos y sociales dan la pauta para cuestionar si el estado de Tamaulipas está preparado para recibir la apertura de la industria extractiva, y amortiguar los posibles impactos sociales, ambientales y económicos que traerá consigo esta actividad industrial. Es preponderante cuestionarse si es posible hablar de un desarrollo sustentable en la región, o de una (in)sustentabilidad a la que posiblemente se esté encaminando la entidad.

\section{Exploración y explotación de gas en lutitas en Tamaulipas}

El estado de Tamaulipas en México forma parte de dos activos de explotación y exploración de hidrocarburos, uno es el Activo Integral Burgos localizado en el Noreste de México (comprende parte de los estados de Tamaulipas, Nuevo León y Coahuila); y otro el Activo de Producción Poza Rica-Altamira, en la provincia Tampico-Misantla, (comprende la parte sur del estado de Tamaulipas y parte del territorio de Veracruz). Dentro de los objetivos de estos activos está el Proyecto Integral Burgos de donde se pretende incrementar la oferta nacional mediante la explotación de campos con reservas probadas de gas no asociado al petróleo más importante del país (Pemex gas y Petroquímica básica). Estudios realizados por Petróleos Mexicanos (Pemex) han logrado documentar que estos activos tienen potencial para el desarrollo de energías renovables, así como para el aprovechamiento de yacimientos no convencionales contenidos de gas en lutitas [4].

La producción de gas asociado del Activo Integral Burgos en 2014 representó el 1.39 mientras que la producción de gas no asociado el 67.39 por ciento del total del país. En este sentido, la entrada de la inversión privada en la industria petrolera en México permitiría la explotación de hidrocarburos en este activo de manera intensiva donde el gas en lutitas es el hidrocarburo con mayor presencia (Pemex, 2013 y U.S. EIA, 2013). 


\section{La exploración y explotación de gas en lutitas}

No obstante, éste se encuentra en yacimientos no convencionales que requieren de la técnica del fracking (fracturación hidráulica) para su extracción, misma que ha sido catalogada como altamente contaminante y con repercusiones en el ambiente y la población aledaña a los sitios de extracción.

Del total de municipios del estado de Tamaulipas, 14 de ellos han sido ubicados en el Activo Integral Burgos y con potencial para la explotación y extracción de este tipo de gas. Estos municipios son: San Fernando, Reynosa, Guerrero, Miguel Alemán, Mier, Camargo, Díaz Ordaz, Río Bravo, Méndez, Valle Hermoso, Nuevo Laredo, Matamoros, Cruillas y Burgos.

Cabe destacar que, para facilitar la entrada de la inversión privada y hacer posible la exploración y explotación de los hidrocarburos en el país, se aprobó en el año 2013 la Reforma Energética la cual funge como el instrumento político que facilitaría la apertura de la industria petrolera en México. Las principales modificaciones realizadas en la Constitución Política de los Estados Unidos Mexicanos en materia de energía quedaron estipuladas en la edición del Diario Oficial de la Federación, publicado el viernes 20 de diciembre de 2013, quedando modificados los artículos 25, 27 y 28 . Sin duda, estas modificaciones son el instrumento que facilita la transición de la industria petrolera en el país. Sin embargo, más allá de los diversos beneficios que el gobierno ha enunciado en términos de desarrollo para el país, los beneficios sociales no han sido explícitos ni se enfatiza sobre de ellos.
A fin de revertir la falta de claridad en dichos puntos, el gobierno estatal de Tamaulipas formuló la denominada Agenda Energética de Tamaulipas, la cual tuvo como como objetivo "prever acciones, conjuntar esfuerzos y asegurar que las inversiones que se realicen en este sector respeten criterios de sustentabilidad y se vean reflejados en un incremento en la calidad de vida de los habitantes del estado" (Gobierno del Estado de Tamaulipas, s.f., p. 32). De manera esquemática, dicha agenda atiende una serie de prioridades estructuradas en tres grupos: 1) capital humano, desarrollo económico e integración social; 2) infraestructura, ordenamiento territorial, sustentabilidad y administración de riesgos; y, 3) marco institucional. Además, busca reafirmar la congruencia entre las actividades relacionadas del sector energético del estado y la política energética del Gobierno Federal. De ésta, se espera que el auge energético en el estado produzca un incremento poblacional, principalmente migratorio, que demandará más y mejores servicios públicos e infraestructura urbana planeada. Sin embargo, dentro de su estructuración surgen diversas interrogantes sobre los beneficios tangibles que obtendrán los habitantes de la entidad.

Con respecto a la política ambiental y sustentabilidad, la Agenda Energética de Tamaulipas resulta poco clara sobre la manera en que el estado hará frente a la posible problemática ambiental que traerá consigo la exploración y explotación de hidrocarburos. En uno de 


\section{La exploración y explotación de gas en lutitas}

sus apartados, se menciona la creación del Fondo Ambiental el cual contempla "un capítulo especial para el resarcimiento de impactos de la industria petrolera y energética, y de impulso a proyectos energéticos de alto impacto social". No obstante, no se especifica en qué consiste este fondo, cómo se aplicará, y qué beneficios tendrá la sociedad.

\section{Fracking. Efectos ambientales}

La extracción de gas en lutitas se realiza en yacimientos no convencionales donde, y a diferencia de los yacimientos convencionales, el consumo de agua es muy alto y el costo de extracción es elevado. La extracción de este tipo de gas requiere del uso de la técnica del fracking. Esta técnica implica hacer una perforación vertical, debido a que los yacimientos se encuentran a una mayor profundidad en comparación con los yacimientos convencionales. Posterior a ello, se realiza una perforación horizontal para tener una mayor cobertura en la explotación de gas de esquisto. Esta técnica requiere de la inyección de grandes volúmenes de agua, arena, y de otros agentes químicos a alta presión para extraer el gas contenido en las rocas (Campero, 2014; Estrada, 2013).

La aplicación de esta técnica, de acuerdo con la experiencia internacional, requiere de grandes volúmenes de agua, pero esta puede variar dependiendo de la zona en función de la permeabilidad de la formación rocosa, la longitud y la profundidad del pozo.
En Estados Unidos, 1a U.S. Environmental Protection Agency (U.S. EPA) estimó que se utilizan entre 70140 billones de galones de agua para fracturar 35,000 pozos cada año. Esto es aproximadamente el consumo anual de agua de 40 a 80 ciudades, cada una con una población de 50,000 habitantes (Earthworks, Hydraulic Fracturing 101, 2014), mientras que, para países como España, se estima un consumo de entre 9,000 y 29,000 metros cúbicos en un pozo (CSCO y SMA, 2014, p.14). A grosso modo, Estrada (2013) menciona que la explotación en yacimientos no convencionales, en comparación con la perforación convencional, requiere grandes volúmenes de agua -alrededor de diez veces más -. Por su parte, Campero (2014, p. 42) afirma que "...el consumo de agua puede ser de 9 a 29 millones de litros por pozo (...) apunta que, si abriéramos 20 mil pozos, estaríamos requiriendo el agua necesaria para uso doméstico y de uso personal anual que utilizan entre 5 y 15.9 millones de personas".

Los impactos negativos que produce el fracking, además de la amplia literatura existente sobre éstos en el ambiente y la sociedad, son múltiples. Por ejemplo, Estados Unidos uno de los principales productores de gas en lutitas en el mundo, ha presentado diversos problemas socioambientales derivados de la aplicación de la técnica. Wright et al. (2012) señala que el agua subterránea cerca de Wyoming contenía sustancias químicas asociadas con esta técnica. Menciona que en el acuífero se detectaron productos químicos sintéticos, como glicoles y alcoholes, compatibles con la producción de gas y fluidos de fractura hidráulica. 


\section{La exploración y explotación de gas en lutitas}

En otros casos se han encontrado diferentes sustancias en el agua como benceno (Di Giulio et al., 2011), bromuros, materiales radioactivos como uranio, radio y radón (Resnifoff et al., 2010) y filtraciones de metano (Osborn et al., 2011), todos ellos provenientes del proceso de extracción de gas en lutitas.

En Argentina, tercer lugar a nivel mundial con reservas recuperables de gas de lutitas, la extracción ha sido acompañada por acciones públicas y comunitarias en contra de la aplicación de esta técnica, incluyendo acciones legales orientadas a frenar su uso.
Por ejemplo, la firma de contratos entre el gobierno nacional y la empresa Chevron, y entre Chevron y la recientemente estatizada YPF (Yacimientos Petrolíferos Fiscales) generaron manifestaciones y situaciones de conflicto público, incluyendo represión policial en diversas zonas de Neuquen. En este lugar, se ubica el mayor depósito de gas en lutitas del país, y, por tanto, un gran interés público y privado para su explotación, particularmente, en la reserva que lleva el nombre de Vaca Muerta (Taillant et al, 2013).

Figura No 1

Implicaciones ambientales del fracking

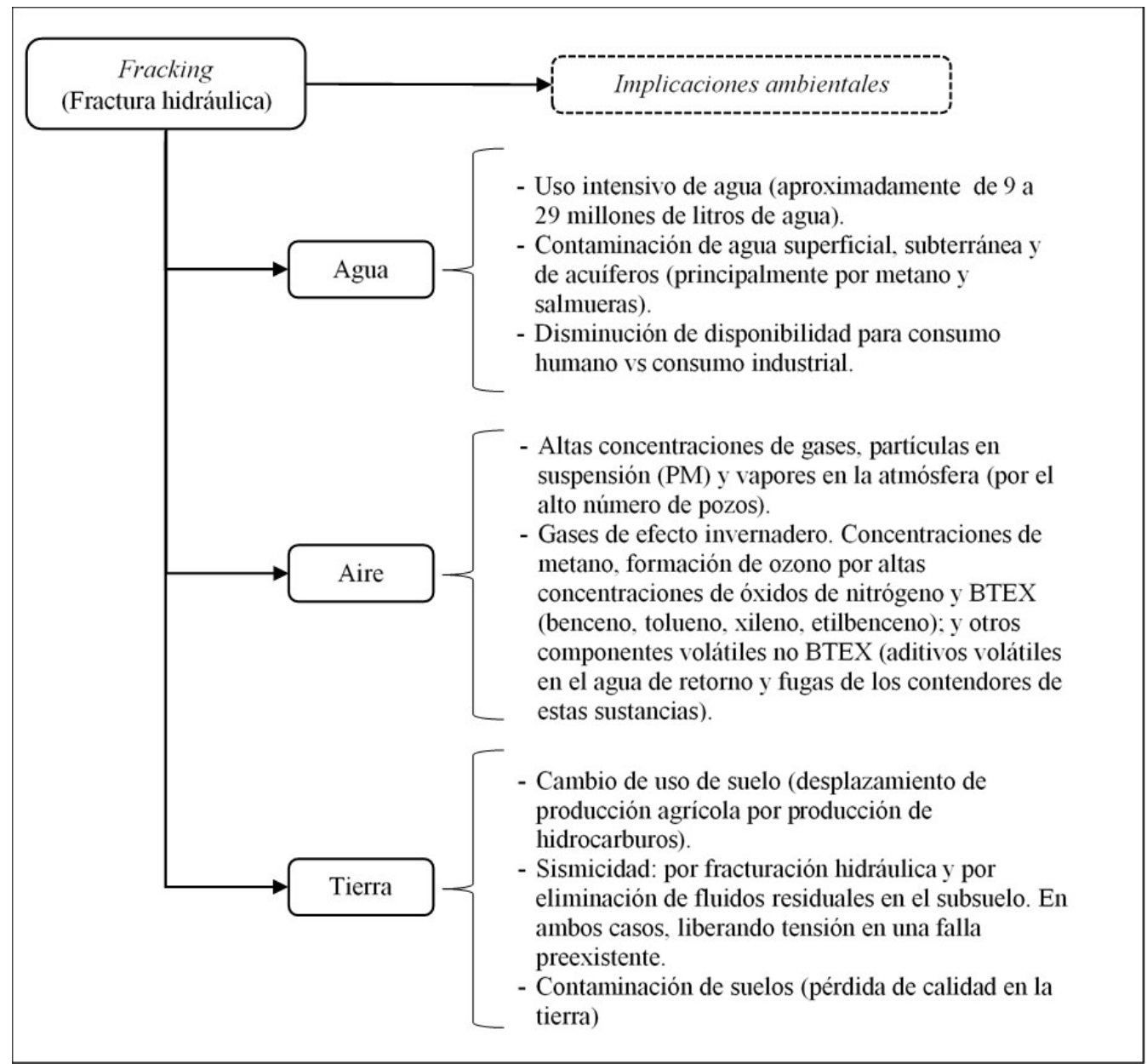

Fuente: Elaboración propia con información de Taillant, J.D., Roeloffs, A., y Headen C. (2013); Alianza Mexicana contra el Fracking (2013); García de Domingo, A., Ponce de León Gil, D., Vadillo Fernández, L. (2014) y Campero, Arena C. (2014). 


\section{La exploración y explotación de gas en lutitas}

En España no hay ninguna normativa nacional que regule el uso de la fractura hidráulica, y el Gobierno ha rechazado prohibirlo a nivel estatal, son las comunidades autónomas las que están publicando sus propias regulaciones. En países como Francia, Bulgaria, Suiza, Australia, Canadá, Italia, Nueva Zelanda, Reino Unido, Rumanía, Sudáfrica, Alemania, Republica Checa, Irlanda del Norte e Irlanda se han establecido prohibiciones y moratorias con respecto a uso de la técnica de fracking para la explotación de gas en lutitas.

La experiencia internacional da indicios de que los posibles beneficios económicos esperados con la extracción de gas en lutitas, más que favorecer a la población, traerá consigo serios problemas socioambientales, en donde los únicos beneficiados serán las empresas privadas que inviertan en el país, mientras que la población solo recibirá los impactos negativos de esta actividad industrial.

De manera esquemática, la anterior figura presenta algunas de las diversas implicaciones ambientales en el agua, aire y tierra como consecuencia de la utilización del fracking.

La evidencia presentada previamente, pone en cuestionamiento los alcances que podría tener la Agenda Energética de Tamaulipas en términos de impactos sociales y ambientales, y por ende en términos de un desarrollo sostenible, una vez que inicien los trabajos de extracción de hidrocarburos, razón por la cual daría la impresión de que Tamaulipas se encamina hacia una (in)sustentabilidad. En el apartado posterior se profundiza en este punto, tomando como ejemplo el caso del agua.

\section{Explotación mediante el fracking en Tamaulipas y sus efectos en el agua}

El norte del país se caracteriza por su baja disponibilidad de agua, mientras que en el año 2012 en Tamaulipas existía la posibilidad de sequía extrema para el año 2015 presentó sequía moderada debido a que se presentaron precipitaciones sobre la media (CONAGUA, 2013 y 2016). En el año 2015, el volumen de agua concesionado fue de 4, 215.1 millones de metros cúbicos, lo cual representa el 4.9 por ciento del total del país. Los usos del agua en el estado corresponden en mayor medida al uso agrícola con un 88 por ciento y al abastecimiento público con un 7.9 por ciento. Ante la baja disponibilidad de agua y al hecho de que la mayor parte es utilizada por el sector agrícola, resultan alarmantes los grandes volúmenes de agua requerida por esta técnica, como también evadir la responsabilidad de las futuras implicaciones que se tendrá con respecto a la disponibilidad futura de agua para el consumo humano y del ecosistema, aunado a la baja calidad del recurso agua que se regresará. Por ello es preponderante preguntarse en este punto ¿De dónde saldrá el agua requerida para la aplicación del fracking en el estado de Tamaulipas? ¿Qué medidas contempla el gobierno estatal para hacer frente a la poca disponibilidad de agua? Este punto no está resulto en la Agenda Energética de Tamaulipas, solo menciona en uno de sus puntos la elaboración de "estudio de demanda del recurso agua por sectores sectores productivos e identificación de estrategias de abasto, - 


\section{La exploración y explotación de gas en lutitas}

uso y reúso del recurso considerando las demandas del sector energético" pero no enfatiza en garantizar el derecho humano al agua para la sociedad tamaulipeca como una prioridad.

\section{Implicaciones en la sustentabilidad de Tamaulipas}

La entrada de la inversión privada en el estado de Tamaulipas implica el cambio de usos de suelo, es decir, la producción agrícola se sustituye por la producción de hidrocarburos, en donde, a través de la creación Ley de Hidrocarburos, estipulada en la edición del Diario Oficial de la Federación publicado el día 11 de agosto de 2014, en el capítulo IV, artículos 100 al 117, se concierta el uso de "suelo y ocupación superficial" para la exploración y explotación de hidrocarburos en zonas con probables reservas, con esta ley el gobierno legítima el cambio de uso de suelos de producción agrícola a producción de hidrocarburos. En este sentido y retomando a D. Barkin (1998, p.3) quien menciona "la sostenibilidad no es posible en las áreas rurales de Latinoamérica mientras la expansión del capital aumente los rangos de pobreza e impida el acceso de los pobres a los recursos necesarios para la mera sobrevivencia [...] el mercado sigue desplazando a la gente a abandonar sus comunidades, empobreciéndola y sus entornos". Este desplazamiento de la población que posiblemente se de en los municipios del estado con reservas de gas en lutitas, solo reafirma la separación de la sociedad de sus medios naturales de reproducción, imposibilitando la generación de un desarrollo sustentable en términos de bienestar social.
Dentro de la estructuración del concepto desarrollo sustentable se mencionó que la pobreza es la que genera la degradación ambiental, en este sentido Martínez-Alier (1992,p. 101) en su conceptualización del ecologismo de los pobres menciona que "la riqueza es una mayor amenaza para el ambiente que la pobreza. Cuando la economía crece, utiliza más recursos naturales y produce más residuos. Por tanto, la tesis de que el ecologismo tiene raíces sociales que surgen de la prosperidad, se podría plantear precisamente en términos de una correlación entre riqueza y producción de desechos y agotamiento de recursos". De acuerdo con esto, el impacto negativo que tendrá la industria petrolera en el estado de Tamaulipas con la utilización de la técnica del fracking, solo profundizará la problemática ambiental, por ejemplo, agudizará la escasez de agua que existe en el estado; donde el derecho humano al agua debe ser una prioridad.

En este sentido la privatización de la industria del petróleo en México obedece a lo que D. Harvey denomina "acumulación por desposesión", concepto acuñado en su obra The New Imperalism, el cual implica la acumulación primitiva u originaria que planteaba Marx en El Capital, en el cual;

"la acumulación originaria incluye la mercantilización y privatización de la tierra y la expulsión forzosa de las poblaciones campesinas; la conversión de diversas formas de derechos de propiedad -común, colectiva, estatal, etc.- en derechos de propiedad exclusivos; la supresión del derecho a los bienes comunes; la transformación - 
Figura 2. Impactos generados por la explotación y exploración de gas en lutitas

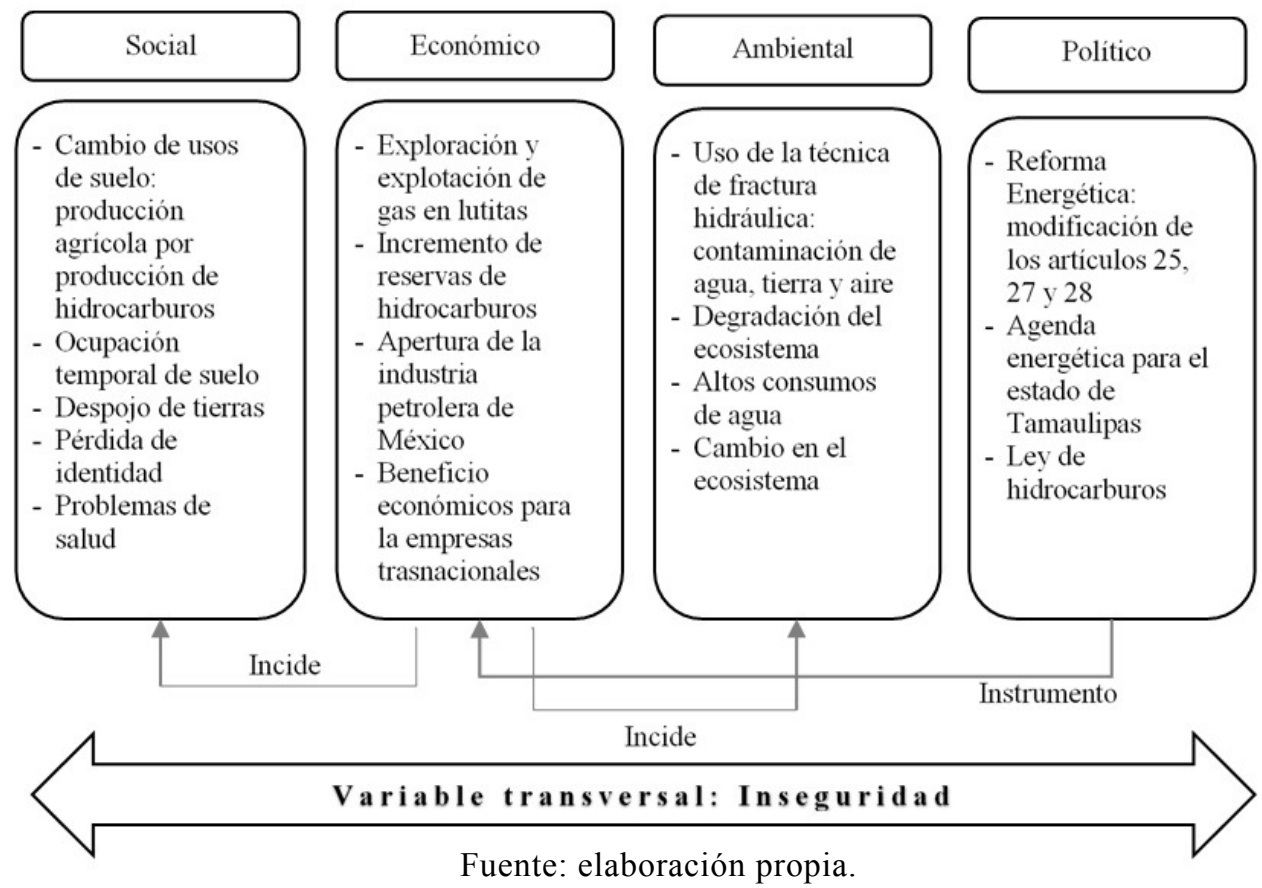

En este sentido, la aplicación del concepto de desarrollo sustentable debería reorientar las actividades económicas acordes a las necesidades de la sociedad con la menor degradación posible del planeta, así como la de generar las condiciones idóneas para el bienestar social y ambiental.

Desde la perspectiva de un desarrollo sustentable radical plateado por $\mathrm{P}$. Burkett (2006), quien destaca la conceptualización de la sustentabilidad basada en términos marxistas, y que implica una gestión adecuada de los recursos naturales, es decir, a los ritmos que la propia naturaleza marca y no a los ritmos que el crecimiento económico ha marcado a lo largo de la historia económica. Además, de reorientar las actividades económicas acordes a las necesidades de la sociedad con la menor degradación posible del planeta, así como la de generar las condiciones idóneas para el bienestar de la sociedad y el ambiente en conjunto.

\section{Conclusiones}

El marcado crecimiento en los últimos años en la producción de gas en lutita en el mercado estadounidense llevó a realizar estudios sobre la disponibilidad de reservas recuperables en el mundo, en los cuales figura de manera preponderante México. Las reformas estructurales en los últimos años, de manera específica la referente a materia energética, dan la pauta a la apertura de la inversión privada para la extracción de este recurso natural, misma que presenta ambigüedad con respecto al proceso en el que se hará la transición, y sobre las herramientas y métodos con los que enfrentarán los diversos problemas sociales y ambientales que deriven de esta apertura. 


\section{La exploración y explotación de gas en lutitas}

De manera general se concluye que es poco visible percibir la incorporación de un desarrollo sustentable en estado de Tamaulipas, que más bien se encamina a una (in)sustentabilidad en donde los múltiples beneficios económicos de la apertura de la industria extractiva en el país no compensarán los impactos negativos que se pueden ocasionar por el uso de la técnica del fracking, los cuales provocarán externalidades negativas tanto a la sociedad como al medio ambiente. El estado de Tamaulipas deberá estar preparado para hacer frente a los problemas de disponibilidad y contaminación de agua, suelo y tierra, y a los posibles efectos sociales que de éstos se derivarán (por ejemplo, profundización del rezago social, despojo temporal de las tierras, demanda insatisfecha en términos de infraestructura y de servicios en general).

Finalmente, es necesario exponer alternativas de análisis para estudiar los diferentes impactos sociales y ambientales que derivarán de esta actividad industrial, siendo preponderante la inclusión de la participación social para un diseño adecuado de propuestas que deriven en un beneficio social y ambiental más que económico.

\section{Bibliografía}

Alianza Mexicana contra el Fracking (2013). "Principales problemas identificados con la explotación de gas de esquisto por fractura hidráulica en México (fracking)". México: FUNDAR. http://fundar.org.mx/mexico/pdf/Documen toFrackingMexico.pdf Octubre 2014

Barkin, David (1998). Riqueza, pobreza y desarrollo sustentable, México: Editorial Jus y Centro de Ecología y Desarrollo.
Barkin, David (2008). "Presentación" en Argumentos. Estudios críticos de la sociedad, Vol. 21, No. 56, pp. 7-15. BP Statistical Review of World Energy. June 2014. Recuperado de http://www.bp.com/en/global/corporate/ about-bp/energy-economics/statisticalreview-of-world-energy.html Octubre 2014

Burkett, Paul (2006), Marxism and Ecological Economics. Toward a red and green political economy. Netherlands: Brill.

Burkett, Paul (2008). "La comprensión de los problemas ambientales actuales vistos con el enfoque marxista" en Argumentos. Estudios críticos de la sociedad, Vol. 21, No 56, pp. 21-32.

Campero, Arena C. (2014). "Impactos socioambientales en los procesos de fractura hidráulica". En Benjamín Robles Montoya (Coordinador). Impacto social y ambiental del fracking. México: Senado de la República, LXII Legislatura, Instituto Belisario Domínguez, y Alianza Mexicana contra el fracking, pp. 41-47.

CONAGUA. (2013). Estadísticas del Agua en México, Edición 2013. México: CONAGUA.

CONAGUA. (2016). Estadísticas del agua en México, Edición 2016. México: CONAGUA.

Confederación Sindical de Comisiones Obreras (CSCO) y Secretaría de Medio Ambiente (SMA). (2014). Impacto ambiental del sistema de fracturación hidráulica para la extracción de gas no convencional. Madrid, pp. 14. 


\section{La exploración y explotación de gas en lutitas}

Recuperado de https://info.nodo50.org/IMG/ pdf/informe fracking.pdf 19 de enero de 2014

Diario Oficial de la Federación, 20 de diciembre de 2013. Recuperado de http:// www.dof.gob.mx/nota_detalle.php? $\operatorname{codig}_{0}=5327463 \&$ fecha $=20 / 12 / 2013$ Octubre 2014

DiGiulio, Dominic, Wilking, Richard, Miller, Carlyle y Oberley, Gregory (2011), "Investigation on Ground Water Contamination near Pavillion, Wyoming, Ada: Environmental Protection Agency". Recuperad o de http://www2.epa.gov/sites/ production/files /documents/ EPA_ReportOnPavillion_Dec-8-2011.pdf Octubre 2014

Earthworks, Hydraulic Fracturing 101. [19 de enero de 2014]. Recuperado de http:// www.earthworksaction.org/issues/de tail/ hydraulic_fracturing_101\#.UkwHMtL IbK0

Estrada, Javier (2013). Desarrollo del gas lutita (shale gas) y su impacto en el mercado energético de México: reflexiones para Centroamérica. México: CEPAL México/ Naciones Unidas.

Foladori, Guillermo y Humberto Tommasino (2000), "El concepto de desarrollo sustentable treinta años después" en Desenvolvimento e Meio Ambiente, No. 1, pp. 41-56. Editora da UFPR

García de Domingo, A., Ponce de León Gil, D., Vadillo Fernández, L. (2014). Recomendaciones ambientales en relación con las medidas preventivas y correctoras a considerar en proyectos relacionados con la exploración y explotación de hidrocarburos mediante técnicas de fractura hidráulica. España: Ministerio de Agricultura, Alimentación y Medio Ambiente, Ministerios de Economía y Competitividad e Instituto Geológico y Minero de España.
Gobierno del Estado de Tamaulipas (s.f), Agenda Energética de Tamaulipas, $\mathrm{M}$ é x i c o: $\mathrm{T}$ a $\mathrm{m}$ a u $1 \mathrm{i} \mathrm{p}$ a $\mathrm{s}$ http://indicadorpolitico.mx/images/pdfs/t amaulipas-agenda-energetica.pdf,

[Octubre 2014]

Harvey, David (2004), "El "nuevo" imperialismo: acumulación por desposesión", en Socialist register 2004, Buenos Aires: CLACSO. pp. 99-129

Independent Statistics \& Analysis/ U.S Energy Information Administration (Junio 2013). Technically recoverable shale oil and shale gas resources: An assessment of 137 shale formations in 41 countries outside the United States. Washington, DC 20585 : U.S. Department of Energy.

Instituto Nacional de Estadística y Geografía (INEGI) (2010), Censos de población y vivienda 2010, México, INEGI.

Ley de Hidrocarburos. Diario Oficial de la Federación, 18 de agosto de 2014.

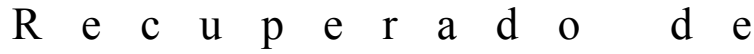
http://www.diputados.gob.mx/LeyesBib lio/pdf/LHidro_110814.pdf

Martínez Alier, Joan (1992), De la Economía Ecológica al Ecologismo Popular. Barcelona: Icaria

Meadows, Donella H.,Dennis L. Meadows, Jorgen Randers y William W. Behrens III (1972), The limits to growth. A report for the Club of Rome's project on the predicament of mankind. New York: Universe Books.

Osborn, Stephe, Vengosh, Avner, Warner, Nathaniel y Jackson, Robert (2011), "Methanecontamination of drinking water accompanying gas-well 


\section{La exploración y explotación de gas en lutitas}

drilling and hydraulic fracturing". Recuperad ode http:/www.pnas.org/content/ early/2011/05/ 02/1100682108.full.pdf + html Octubre 2014

Petróleos Mexicanos (PEMEX). (2013). Anuario estadístico PEMEX 2013. México: PEMEX/ Dirección Corporativa de Finanzas.

Petróleos Mexicanos (PEMEX). Base de D a tos Institucional (B D I) http://ebdi.pemex.com/bdi/bdiController.do? action $=$ temas $\&$ fromCuadros $=$ true $]$

Programa de las Naciones Unidas para el Desarrollo en México (2014). Índice de desarrollo humano municipal en México: Nueva metodología. México: PNUD.

Programa de las Naciones Unidas para el Desarrollo en México (2015), Índice de Desarrollo Humano para las entidades federativas, México 2105. Avance continúo, diferencias persistentes. México: PNUD

Resnikoff, Marlin, Alexandrova, Ekaterina y Travers, Jackie. (2010). Radioactivity in Marcellus Shale, Nueva York: Radioactive Waste Management Associates. Recuperado de

http://energy.wilkes.edu/PDFFiles/Library/ Marcellus\%20Shale\%20Radioactivity\%20R eport $\% 205-18-2010 . p d f$

Sachs, Ignacy (1980), "Ecodesarrollo. Concepto, aplicación, implicaciones" en Comercio Exterior, Vol. 30, No. 7, México. Pp. 718-725.

Secretaría de Energía (SENER). http://www.sener.gob.mx/ Octubre 2014.

Secretaria de Energía (SENER). Sistema de Información Energética (SIE). http://sie.energia.gob.mx/bdiController.do? action=temas Octubre 2016.
Taillant, Jorge Daniel, Mariana Valls, María Eugenia D'Angelo, Candace Headen y Anna Roeloffs (2013).

Fracking Argentina. Informe Técnico y Legal Sobre la Fracturación Hidráulica en Argentina. Córdoba Argentina: Centro de Derechos Humanos y Ambiente \& ECOJURE.

The National Petroleum Council (NPC). (2007). Unconventional gas. Working Document of the NPC Global Oil \& Gas. July 18, 2007. Paper No. 29

U.S. EIA (U.S Energy Information Administration) (2013), Technically recoverable shale oil and shale gas resources: An assessment of 137 shale formations in 41 countries outside the United States. Washington, DC 20585: U.S. Department of Energy.

U.S. EPA (U.S. Environmental Protection Agency) (2015), Case study analy $\mathrm{s}$ is of the impacts of w ater acquisition for hydraulic fracturing on local water availability, EPA/600/R-1 4 / 179 .

R e c u p e r a d o d e http:// www2.epa.gov/sites/production/fi les/2015-

07/documents/hf_water_acquisition_rep ort_final_6-3-15_508_km.pdf $1 \overline{0} \mathrm{de}$ Mayo de $\overline{2015 .}$

Wright, P.R., McMahon, P.B., Mueller,

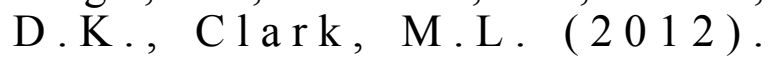
"Groundwater-quality and qualitycontrol data for two monitoring wells near Pavillion". Wyoming, April and May 2012: U.S. Geological Survey Data Series 718, 26 p. Recuperado de http://pubs.usgs.gov/ds/718/ Octubre 2014. 